\title{
Profit Maximization for Service Providers using Hybrid Pricing in Cloud Computing
}

\author{
N.Ani Brown Mary \\ Anna University \\ Tirunelveli, India
}

\begin{abstract}
Cloud computing has recently emerged as one of the buzzwords in the IT industry. Several IT vendors are promising to offer computation, data/storage, and application hosting services, offering Service-Level Agreements (SLA) backed performance and uptime promises for their services. While these 'clouds' are the natural evolution of traditional clusters and data centers, they are distinguished by following a pricing model where customers are charged based on their utilization of computational resources, storage and transfer of data. They offer subscription-based access to infrastructure, platforms, and applications that are popularly termed as IaaS (Infrastructure as a Service), PaaS (Platform as a Service), and SaaS (Software as a Service). In order to improve the profit of service providers we implement a technique called hybrid pricing, where this hybrid pricing model is a pooled with fixed and spot pricing techniques.
\end{abstract}

Keywords: Service-Level Agreements, Infrastructure as a Service, Platform as a Service, Software as a Service, Hybrid Pricing.

\section{INTRODUCTION}

Cloud computing is not a total new concept; it is originated from the earlier large-scale distributed computing technology. However, it will be a subversion technology and cloud computing will be the third revolution in the IT industry, which represent the development trend of the IT industry from hardware to software, software to services, distributed service to centralized service. Cloud computing is also a new model of business computing, it will be widely used in the near future. The core concept of cloud computing is reducing the processing burden on the users' terminal by constantly improving the handling ability of the "cloud", eventually simplify the users' terminal to a simple input and output. All of this is available through a simple Internet connection using a standard browser or other connection. It manages a variety of different workloads, including the batch of back-end operations and user-oriented interactive applications. It rapidly deploy and increase workload by speedy. It provides physical machines or virtual machines. It supports redundancy, self-healing and highly scalable programming model, so that workload can be recover from a variety of inevitable hardware/software failure.

The manufacturing industry is undergoing a major transformation enabled by IT and related smar ttechnologies. Cloud computing is one of such smar ttechnologies. The main thrust of Cloud computing is to provide on-demand computing services with high reliability, scalability and availability in a distributed environment. The National
Institute of Standards and Technology(NIST) [14] defined cloud computing as"a model for enabling ubiquitous, convenient, on-demand network access to a shared pool of configurable computing resources (e.g., networks, servers, storage, applications, and services) that can rapidly provisioned and released with minimal management effort or service provider interaction." Cloud computing provides resources such as processing power, bandwidth and storage capacity. Software as a service providers has to rent resources from the infrastructure as a service providers and provide it to the users, so there is no profitable pricing function for the service providers. So we implement a pricing function called Hybrid pricing to improve the profit of service providers. Here, section II consist of Related Work, section III consist of System Design, section IV,V,VI consists of Fixed, Spot and Hybrid pricing Implementations. At last Section VII consist of Results that has been obtained.

\section{RELATED WORK}

Cloud computing is an emerging technology in the IT world. Some features of cloud, such as low cost, scalability, robustness and availability are attracting large-scale industries as well as small business towards cloud. Cloud computing is a model for enabling convenient, on demand network access to a shared pool of configurable computing resources (e.g., networks, servers, storage, applications, and services) that can be rapidly provisioned and released with minimal management effort or service provider interaction. At present 
some major cloud providers are Amazon Web Services [1], Microsoft Azure [2] and Google AppEngine [3]. These cloud providers offer many type of services for monitoring, managing and provisioning resources and application services. A cloud provider can support more number of users with same number of resources [4]. During the busy hours service provider needs more resources and at other periods of time load on the service providers are very less, so there is a need of continuous scale up and scale down the service providers infrastructure of resources. These scale up and scale down operations require dynamic provision [6].

Yi et al. give an approach to minimize the costs of computations using Amazon EC2s spot instances for resource provisioning. This paper also considers the application of market oriented mechanisms in [7]. Fabien Hermenier et al. proposed a new approach, Entropy, in a homogeneous cluster environment, which takes into account both the problem of allocating the virtual machines to available nodes and the problem of how to migrate the virtual machines to these nodes. The performance overhead is determined by the time required to choose a new configuration and the time required to migrate virtual machines according to the configuration. The Entropy resource manager can choose migrations that can be implemented efficiently, incurring a low performance overhead in [10]. Wei et al. used game theory to handle the resource allocation in cloud computing. In their approach, a Binary Integer Programming method is proposed to solve the parallel tasks allocation problem on unrelated machines connected across the Internet. Their algorithms take both optimization and fairness into account and provide a relatively good compromise resource allocation. But these methods can only be used for seeking optimal allocation solution for the complex and dynamic problems that can be divided into multiple cooperative subtasks in [13].

\section{SYSTEM DESIGN}

When two or more pricing joined together it is called Hybrid pricing. Here fixed and spot pricing are combined to form the Hybrid pricing technique. IaaS providers maintain the virtual machine with the help of the cloud storage. SaaS providers rent resources from the IaaS providers and provide it to the users. Figure 1 Shows clearly the cloud environment consist of virtual machines and it all maintained in the cloud storage and that was controlled by the controller storage. A SaaS provider rents resources from IaaS providers and leases software as services to users. SaaS providers aim at minimizing their operational cost by efficiently using resources from IaaS providers, and improving Customer Satisfaction Level (CSL) by satisfying SLAs, which are used to guarantee QoS requirements of accepted users. From SaaS provider's point of view, there are two layers of SLA with both users and resource providers.. It is important to establish two SLA layers, because SLA with user can help the SaaS provider to improve the customer satisfaction level by gaining users' trust of the quality of service; SLA with resource providers can enforce resource providers to deliver the satisfied service. If any party in the contract violates its terms, the defaulter has to pay for the penalty according to the clauses defined in the SLA.

An IaaS provider, offers VMs to SaaS providers and is responsible for dispatching VM images to run on their physical resources. The platform layer of SaaS provider uses VM images to create instances. It is important to establish SLA with a resource provider, because it enforces the resource provider to guarantee service quality. Furthermore, it provides a risk transfer for SaaS providers, when the terms are violated by resource provider. In this work, we do not consider the compensation given by the resource provider because $85 \%$ resource providers do not really provide penalty enforcement for SLA violation currently [22].

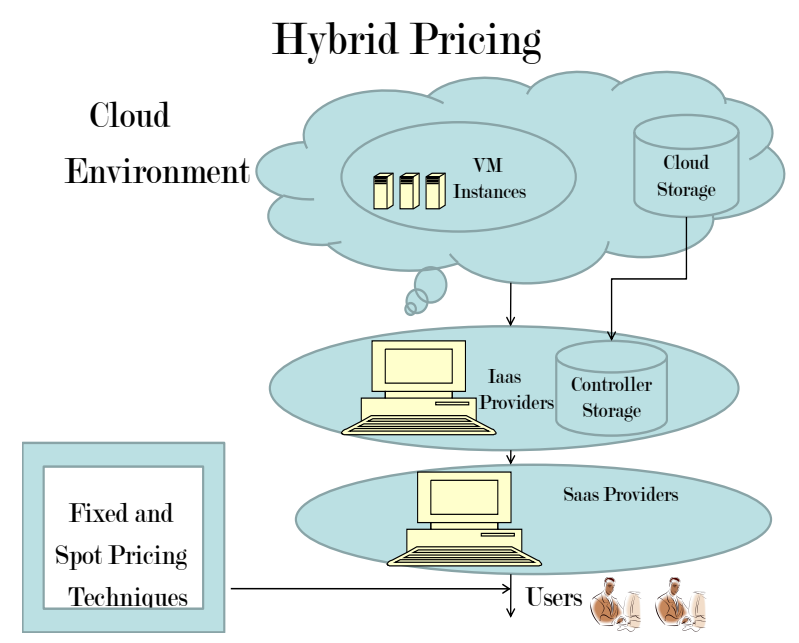

Figure 1: Architecture for Hybrid Pricing

Users and the providers negotiate for the services, that is they have an agreement between them. After having their agreement, resources will be provided to users with the help of the Service Level Agreement contract. If the request can be accepted, a formal agreement (SLA) is signed between both parties to guarantee the QoS requirements such as response 
time. That contract has been explained clearly in figure 2 . The Service Level Agreement includes the following constraints Service Initiation Time that gives how long it takes to deploy a VM. Then Price shows how much a SaaS provider has to pay per hour for using a VM from a resource provider. Then Input Data Transfer Price shows how much a SaaS provider has to pay for data transfer from local machine to resource provider's VM. Then Output Data Transfer Price shows how much a SaaS provider has to pay for data transfer from resource provider's VM to local machine. Then Processing Speed shows how fast the VM can process. Then Data Transfer Speed shows how fast the data is transferred and it depends on the location distance and also the network performance.

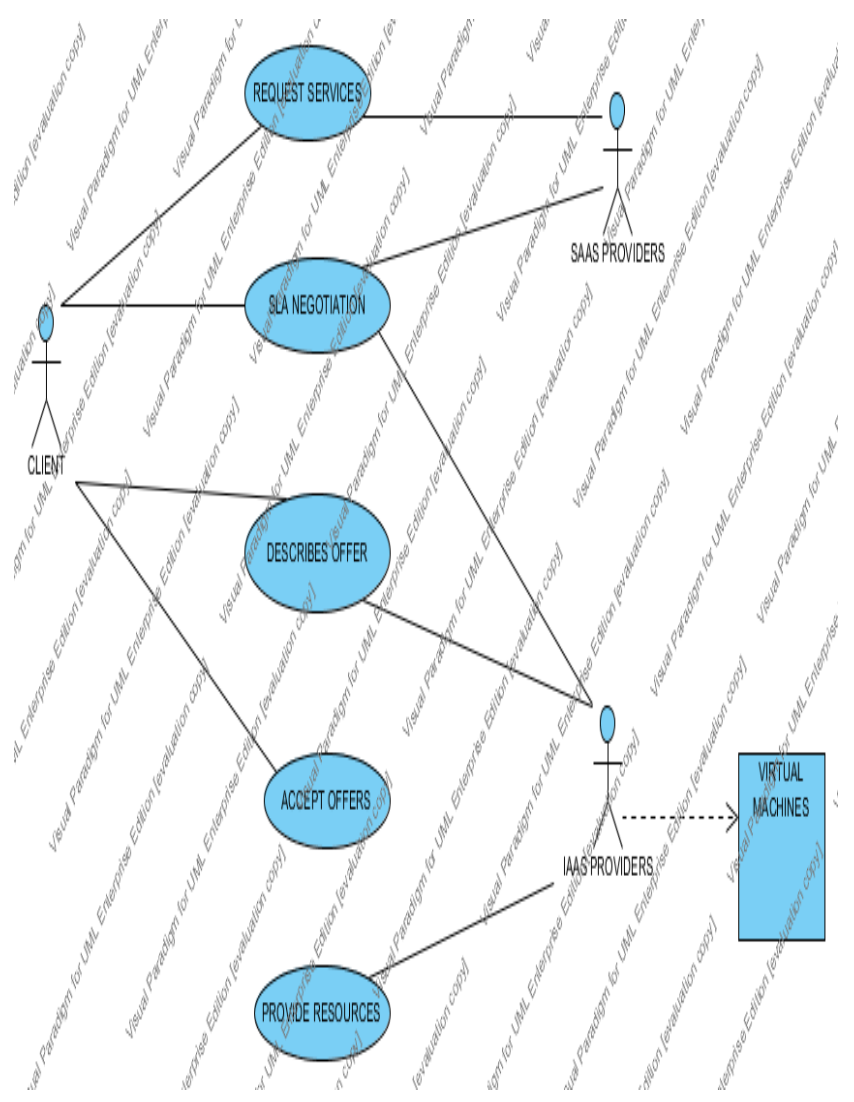

Figure 2: SLA contract

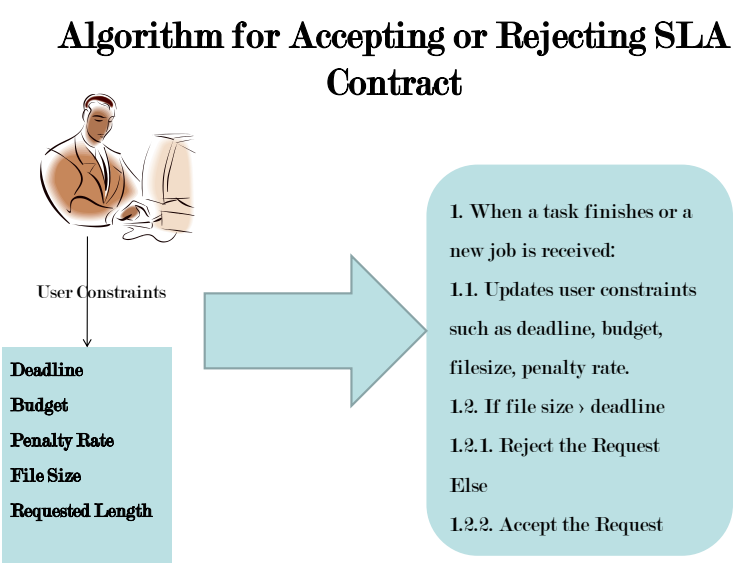

This algorithm depends on user accept or reject the offer provided by IaaS providers. Here cost and profit are calculated. If there is profit for SaaS providers, request will be accepted or rejected. For one second only thousand million instruction per second can be calculated. So users deadline is compared with the file size, if file size is more than the deadline then request will be rejected or it will be accepted. Thus the SLA contract will be either accepted or rejected with the help of the constraints.

\section{FIXED PRICING IMPLEMENTATION}

If the Investment Return is greater than the Expected Investment Return then the resources will be provided. Only fixed price will be offered for users. Here we get users five constraints they are deadline, budget, penalty rate ratio, input file size and requested length. Deadline first shows the maximum time user would like to wait for the result. Then the Budget shows how much user is willing to pay for the requested services. Then Penalty Rate Ratio shows ratio for consumers' compensation if the SaaS provider misses the deadline. Then Input File Size asks the size of input file provided by users. Then Requested Length shows how many Millions of Instructions (MI) are required to be executed to serve the request.

In fixed pricing, we calculate the cost with help of processing cost + data transfer cost + virtual machine initiation cost + penalty delay cost. Then profit for providers is calculated by reducing the cost from the budget that is obtained from users. 


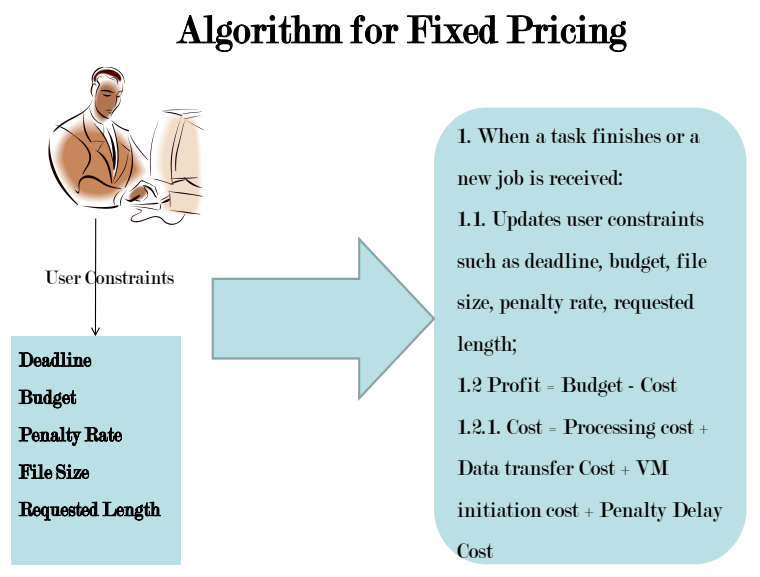

\section{SPOT PRICING}

\section{IMPLEMENTATION}

When more than one users request for the same resource at the same time, depending on the profit of SaaS providers the resource will be provided to the user. This algorithm is based on users given inputs such as Deadline, Budget, File Size. Here Investment Return and Expected Investment Return are calculated. In this technique, response time is calculated by just calculating the processing time, virtual machine initiation time and penalty delay time. Return Investment is calculated by the profit by the response time. Expected Return Investment is calculated by the cost by the response time. The condition is if the Return Investment Return is more than the Expected Investment Return then there is profit for providers so the resources are provided.

\section{Algorithm for Spot Pricing}

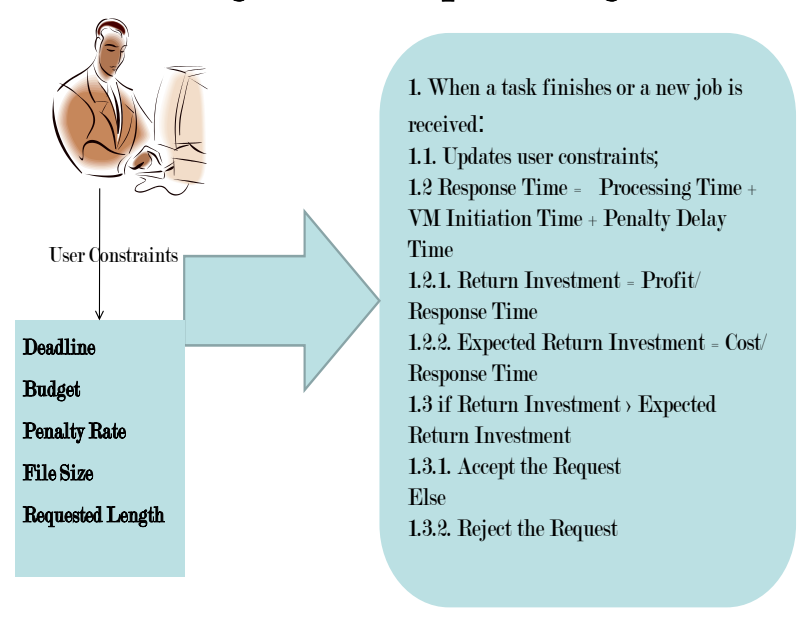

\section{HYBRID PRICING}

\section{IMPLEMENTATION}

Hybrid pricing is a complete combination of fixed and spot pricing. This algorithm is used when more than one user request for the same resources at the same time. Deadline, Budget and File size are obtained by more than one user. The user who provides more profit for SaaS providers will be selected and resources will be provided.

From a SaaS provider's point of view, there is a legal contract-SLA with any customer and if any party violates SLA terms, the defaulter has to pay for the penalty according to the clauses defined in the SLA. The SLA properties include SaaS provider pre-defined parameters and the customer specified QoS parameters. The properties defined in the SLA are as follows, Request Type defines the customer request type, which is 'fist time rent' or 'upgrade service'. 'First time rent' means the customer is the customer who is renting a new service from this SaaS provider. 'Upgrade service' includes two types of upgrade, which are 'add account' and 'upgrade product'. Then Product Type shows the software product offered to customers. Then Account Type constrains the maximum number of accounts a customer can create. Contract Length shows how long the software service is legally available for a customer to use. Number of Accounts shows the actual number of accounts that a customer wants to create. Then Number of Records shows the maximum number of records a customer is able to create for each account during the transaction and this will impact the data transfer time during the service upgrade. Response Time represents the elapsed time between the end of a demand on a software service and the beginning of a service.

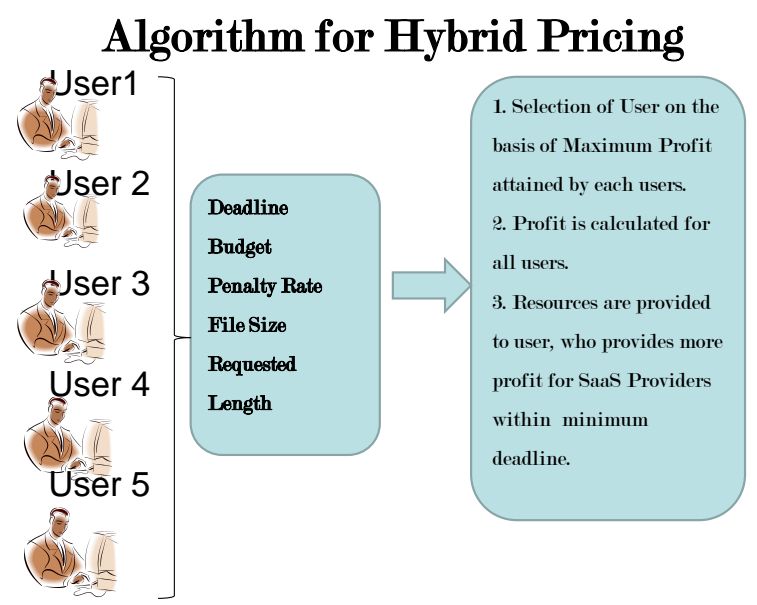


Here the concept of both fixed and spot pricing are combined together to form the hybrid pricing. Since fixed and spot pricing are totally new concept they both are combined together to improve the profit of service providers.

\section{RESULTS}

The results shows the comparison between fixed, spot and hybrid pricing. On comparing with deadline and budget we can see each results clearly shows the profit maximization using hybrid pricing. Totally there are five constraints, here we took two constraints they are deadline and budget. Both are compared with the three pricing techniques.

Table 1: Deadline Vs Profit

\begin{tabular}{|l|c|c|c|}
\hline $\begin{array}{l}\text { Deadline(secs)/ } \\
\text { Profit(Rupees) }\end{array}$ & $\begin{array}{c}\text { Fixed } \\
\text { Pricing }\end{array}$ & $\begin{array}{c}\text { Spot } \\
\text { Pricing }\end{array}$ & $\begin{array}{c}\text { Hybrid } \\
\text { Pricing }\end{array}$ \\
\hline Tight & 1000 & 2200 & 3800 \\
\hline Medium & 1800 & 3400 & 4600 \\
\hline Relax & 2100 & 3600 & 4900 \\
\hline Very Relax & 2900 & 4400 & 5800 \\
\hline
\end{tabular}

This table shows clearly that Hybrid pricing increases the profit compared with the fixed and spot pricing. Tight shows that seconds range between one to six seconds and medium shows the range between six to twelve seconds. Relax shows that the process is performed in very relaxed way and very relax shows that user is waiting for a long time.

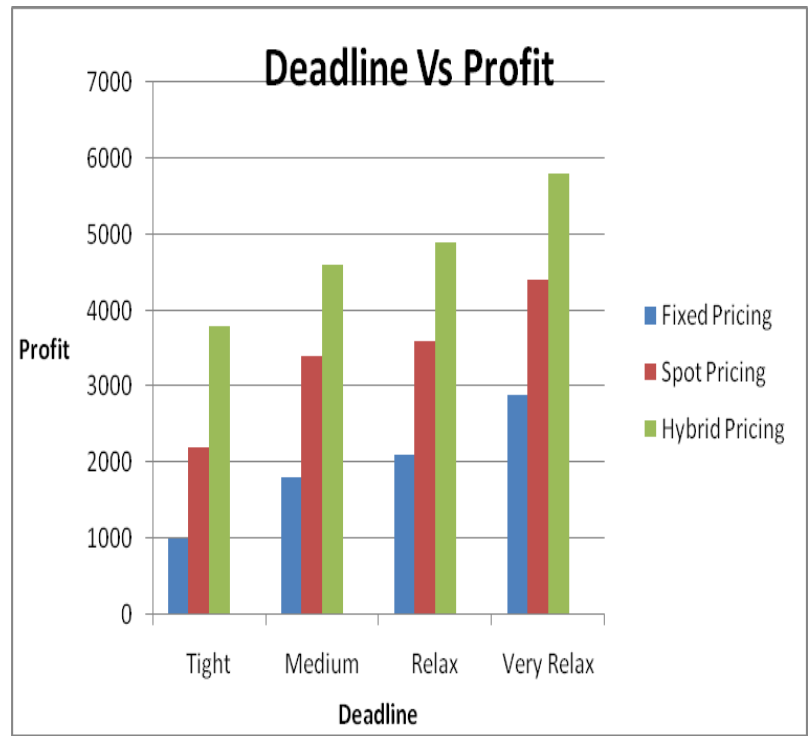

Figure 3: Deadline Vs Profit

This figure shows the profit maximization using hybrid pricing to a value of 6000 compared to the fixed and spot pricing. When compared to tight, very relax provides more profit for service providers. This shows Hybrid pricing provides more profit compared to other pricing techniques.

Table 2: Budget Vs Profit

\begin{tabular}{|l|c|c|c|}
\hline \multicolumn{1}{|c|}{$\begin{array}{c}\text { Budget/ } \\
\text { Profit(Rupees) }\end{array}$} & $\begin{array}{c}\text { Fixed } \\
\text { Pricing }\end{array}$ & $\begin{array}{c}\text { Spot } \\
\text { Pricing }\end{array}$ & $\begin{array}{c}\text { Hybrid } \\
\text { Pricing }\end{array}$ \\
\hline Small & 1245 & 2300 & 3756 \\
\hline Medium & 2600 & 3400 & 4200 \\
\hline Large & 4600 & 5235 & 6344 \\
\hline
\end{tabular}

This table shows that the budget that is provided by users in three ways that is small budget users, medium budget users and large budget users. Here the profit is obtained more in hybrid pricing technique. When compared with fixed pricing, spot pricing gives more profit. When compared with hybrid pricing, this provides more profit.

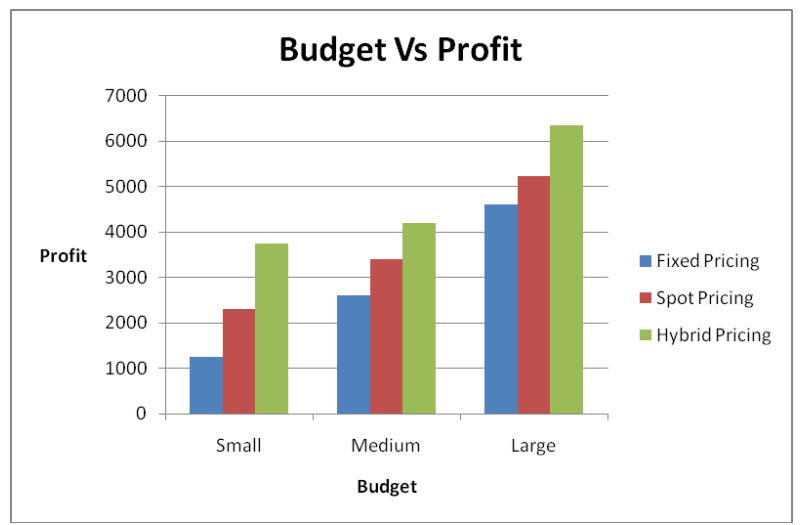

Figure 4: Budget Vs Profit

This graph clearly shows that the profit is maximized in spot pricing compared to the fixed pricing and then profit is more in hybrid pricing compared to the spot pricing techniques.

\section{CONCLUSION}

Thus the main goal to improve the profit of service providers has been satisfied. The three pricing techniques has been explained and implemented and their results are shown. Fixed and Spot pricing both are the best techniques but when they are combined and used it provides more profit when are used single-handedly.

\section{ACKNOWLEDGEMENT}

None of this work would have been possible without the selfless assistance of a great number of people. I would like to 
gratefully thank all those members for their valued guidance, time, helpful discussion and contribution to this work.

\section{REFERENCES}

[1] Amazon Elastic Compute Cloud,"' http://aws.amazon.com'ec2 .

[2] Windows Azure Platform, http://www.microsoft.com.azure/ (March 17,2010).

[3] Google App Engine, http://appengine.google.com(March 17, 2010).

[4] R. Buyya, R. Ranjan, and R. N. Calheiros, "InterCloud: Utilityoriented federation of Cloud computing environments for scaling of application services," in Proceedings of the 10th International Conference on Algorithrns and Architectures for Parallel Processing (ICA3PP'IO), ser. Lecture Notes in Computer Science, vol. 6081. Busan: Springer, May 2010, pp. 13-3.

[5] N.A. Vouk, "Cloud computing-issues, research and implementation", published in Proceedings of 30th International Conference on Information Technology Interfaces(ITI 2008), Dubrovnik, Croatia, 2008.

[6] http://www.vmware.com.virtualization.

[7] Inigo Goiri, Jordi Guitart, Jordi Torres, "Economic model of a Cloud provider operating in a federated Cloud ", Springer Science+Business Media, LLC 2011 Inf Syst Front DOL 10. I007/sI0796-011-9325-x.

[8] P.B. Chun, D.E. Culler, "User-centric performance analysis of market-based cluster batch schedulers", published in Proceedings of the 2nd IEEE/ACM International Symposium on Cluster and Grid Computing (CCGrid 2002), Berlin, Germany, 2002.

[9] Y.C. Lee, C. Wang, A.Y. Zomaya, B.B. Zhou, "Profitdriven service request scheduling in clouds", published in Proceedings of the International Symposium on Cluster and Grid Computing (CCGrid 2010), Melbourne, Australia, 2010.

[10] F. Hermenier, X. Lorca, J.-M. Menaud, G. Muller and J. Lawall. Entropy: a Consolidation Manager for Cluster. In proc. of the 2009 International Conferenceon Virtual Execution Environments (VEE’09), Mar.2009.

[11] C.S. Yeo, R. Buyya, "Service level agreement based allocation of cluster resources: Handling penalty to enhance utility", published in the Proceedings of the 7th IEEE International Conference on Cluster Computing (Cluster 2005), Boston, MA, USA, 2005.

[12] Y.F. Rana, M. Warnier, T.B. Quillinan, F. Brazier, D. Cojocarasu, "Managing violations in service level agreements", published in the Proceedings of the 5th International Workshop on Grid Economics and Business Models (GenCon 2008), Gran Canaria, Spain, 2008.

[13] Guiyi Wei, Athanasios V. Vasilakos, Yao Zheng and Naixue Xiong. A game-theoretic method of fair resource allocation for cloud computing services. The Journal of Supercomputing, Volume 54, Number 2, 252-269.

[14] Mell P, Grance T. Perspectives on cloud computing and standards. National Institute of Standards and
Technology (NIST). Information Technology Laboratory; 2009.

[15] Mario Mac'ias, J. Oriol Fit'o and Jordi Guitart ,"Rulebased SLA Management for Revenue Maximisation in Cloud Computing Markets", published in the Proceedings of the 12th IEEE International Conference on Cluster Computing (Cluster 2009), Boston, MA, USA, 2009.

[16] Hadi Goudarzi and Massoud Pedram ,"Multi-dimensional SLA-based Resource Allocation for Multi-tier Cloud Computing Systems", published in the Proceedings of the International Symposium on Cluster and Grid Computing (CCGrid 2011), Melbourne, Australia, 2011.

[17] Dimitrios Zissis , Dimitrios Lekkas ,"Addressing cloud computing security issue" published in ELESIVER Publications of Future Generation Computer Systems 28(2012) 583-592.

[18] Nir Kshetri ,"Privacy and security issues in cloud computing: The role of institutions and institutional evolution", published in the Proceedings of IEEE International Conference on Service Oriented Computing and Applications (SOCA 2011), Newport Beach, California, USA, 2011.

[19] Dan Svantesson, Roger Clarke ,"Privacy and consumer risks in cloud computing", published in ELESIVER publications of computer law \& security review 26(2010) $391-397$.

[20] Gaofeng Zhang, Yun Yanga, Jinjun Chen, "A historical probability based noise generation strategy for privacy protection in cloud computing", published in ELESIVER Publications in the Journal of Computer and System Sciences 78 (2012) 1374-1381.

[21] Brototi Mondal , Kousik Dasgupta , Paramartha Dutta , "Load Balancing in Cloud Computing using Stochastic Hill Climbing-A Soft Computing Approach", published in ELESIVER publications Procedia Technology 4 ( 2012 ) $783-789$.

[22] CIO, retrieved on 10 Sep. 2010, http://www.cio.com.au. 